\title{
Michael Schuenke, Erik Schulte, Udo Schumacher, Laurence M. Ross, Edward D. Lamperti, Ethan Taub: THIEME atlas of anatomy image collection head and neuroanatomy
}

\author{
Georg Thieme Verlag, Stuttgart, New York, 2007, 1.182 illustrations, 72 tables on DVD, \\ US \$ 199.95, ISBN 13: 978-1-58890-548-2 (TNY), ISBN 13: 978-3 13 144151-5 (GTV)
}

\section{Pierre Kehr}

Received: 16 August 2009 / Accepted: 18 August 2009 / Published online: 1 September 2009

(C) Springer-Verlag 2009

The superb DVD allows for easy and intuitive use. Two headings are available on the left column: Head and Neuroanatomy.

When you click on Head, a tree view control expands to show ten subheadings, which are divided into a great number of sections, from 2 to 21 , depending on the selected item. The user has to move down to the last available section of every chapter to display figures in the large blank area on the right part of the screen. Then you will be pleasantly surprised at the clarity of the figures and diagrams!

Moreover, each page containing figures include excellent tools. An option list is shown on the upper portion with the following functions: « Back »; «With/without label (allowing not to display labels) »; «Copy to clipboard (enabling to copy the clipboard content in 72 dpi, especially useful to insert files into a PowerPoint presentation) »; «Save as ... (for 300 dpi enlargements) »; «Zoom (with three levels of magnification) ».

You will find a keyword "Search" button at the top of the same left column.

Keywords have to be entered in English and the process can seem puzzling at first: for example, if you enter « nasal bone », a great number of sections are listed. These results concern all the items having a connection with the nasal bone. Each section is a hypertext on which the user clicks to view the relevant pages. On the other hand, We deplore that in the DVD, Latin designations are not considered as headwords and are not consistently added to English designations.

Lastly, the visual menu bar located at the very top of the screen features legal information, instructions and an e-mail contact form to reach Thieme Verlag.

Overall, this is a wonderful browsing tool that is also most useful for a presentation including anatomical data (the copyright will be automatically assigned for each copied image).

It is highly recommended to all those who need to browse through general anatomical data or to use this book as a teaching tool.

No funds were received in support of this study.

Pierre Kehr Strasbourg

P. Kehr $(\bowtie)$

Strasbourg, France

e-mail: kehrpier@aol.com 